\title{
The Stress Analysis and Tests on the Hinge Beam of the Diamond Synthesis Cubic Press
}

\author{
Ma Liang ${ }^{1}$, Lu Beihua ${ }^{1}$, Yuan Yuan² and Zhang Yinghong ${ }^{1}$ \\ ${ }^{1}$ School of Mechanical Engineering, Guilin University of Electronic Technology, 541004 Guangxi, China \\ ${ }^{2}$ Guilin Guiye Heavy Industries Co.,Ltd, 541199 Guangxi, China
}

\begin{abstract}
To deal with the problem of the lug fractures of hinge beam caused by the fatigue and overload during the operation of the cubic press, the analysis methods of finite element are applied to the analysis of the internal stress distributions of the hinge beam. The simulation results show that the internal stress of the hinge beam mainly concentrates on the upper surface of the lug roots connecting the outer cylinder with the both lugs. According to the data of simulation and analysis as well as the actual fracture situations, considering the strain-test methods we have designed the schemes of testing the strain on the lugs of hinge beam. And the strain measurements of the lugs are completed by the repeated loading experiments. Comparing the data of simulation and analysis with the measured data has verified their consistency. It also confirms the model established by the simulation and analysis is reasonable and accurate at the same time.
\end{abstract}

\section{Introduction}

Strain gauge measurement [1] is the most common and effective mechanics testing method in the mechanical engineering testing technology. It has not only provided the scientific bases for analyzing the failure causes of the components and optimizing the structure, but also has a good guiding significance for the new product development and modification when we need to take the stress measurement and understand the stress distribution laws of the structures in the safety and reliability analysis of engineering structures.

The cubic press is the prime equipment for compositing the superhard materials in current China[2,3]. The hinge beam, as the key component of the hydraulic press, plays a decisive role in the safety and reliability of the whole hydraulic press system, and founding its internal stress distribution is helpful to improve the structure and performance of cubic press. At present, the analysis of hinge beam is so stagnant in the traditional software analysis based on a large number of assumptions and simplification that the analysis results can not accurately reflect the actual working stress of hinge beam. As it is shown in the Fig. 1 that it is likely to cause some unexpected accidents. Hence, the designed hinge beam of cubic press relying solely on the above analysis method can not accurately mirrors its true stress. Consequently, in order to analyze accurately and effectively the stress and the strain of hinge beam, it is significant for us to provide a set of the complete test schemes and the test data for verifying the theoretical analysis results.
This paper proposes the research of the stress analysis and tests on the hinge beam of the diamond synthesis cubic press. First, 3D design model of the hinge beam is established by ANSYS, a kind of the analysis software of finite element. And carrying out the static analysis and calculation of finite element, we has drawn the equivalent stress distribution nephogram and explored the stress distribution situations of hinge beam. Furthermore, based on the electrical stress-measurement technology [4], the actual tests of the stress distribution of hinge beam are carried out. And comparing the results of finite element analysis with the test data, the both are in the same line, which verifies better rationality and correctness of the established model in this paper.

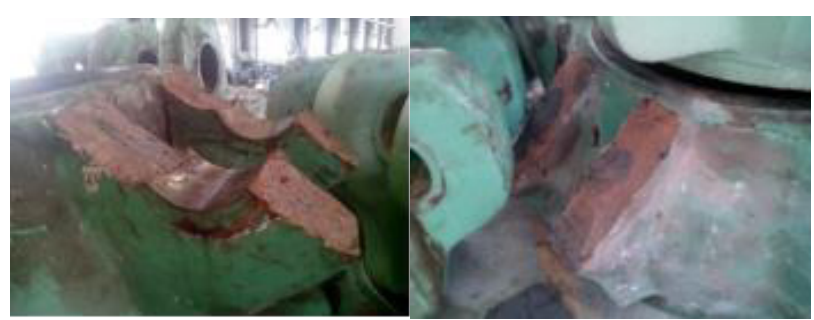

(a) Fractures of Lug Holes (b) Fractures of Lug Roots Figure 1. The Fractures of the Hinge Beam

\section{The framework and working principle of the cubic press}

Main Consisting of the hinge beam, the working block and the piston, the hinged cubic press is an assembly body which is composed of 6 hinge beams through the 
hinged constraints. The Fig. 2 shows the structure of the cubic press.

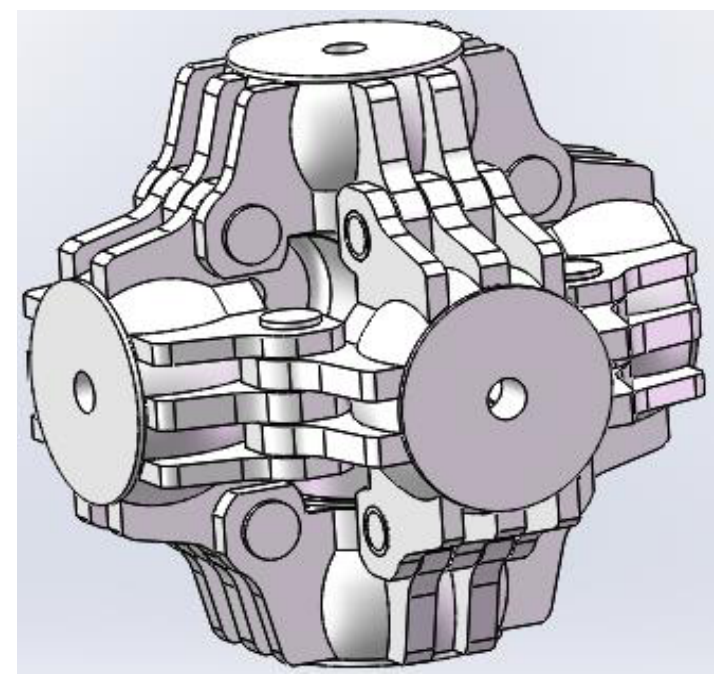

Figure 2. The hinged cubic press

The hinged cubic press is special equipment for producing the synthetic materials on the condition of the high temperature and high pressure [5]. In order to push the piston forward and generate the super-high pressure in the front of the piston, the hydraulic oil is pushed into the center of the main engine from the bottom of six working blocks. Furthermore, with the help of the cemented carbide anvil in the center of the main engine, a super-high pressure chamber with the sealed cube is formed around the raw materials. And then the highpressure conditions on which the diamond and other superhard materials are growing are provided by the pressure the pyrophyllite transfers. At the same time, the high-temperature conditions on which the chambers make the synthetic materials grow are provided by the way of electric heating. After holding pressure and compositing the superhard materials for a period of time on the condition of the high temperature and high pressure, the hydraulic devices removed the anvils again and take out the pyrophyllite from the synthesis chambers, so that the device carry out a list of the operations of the pressurerelief. Finally, the overall operation of compositing the superhard materials is completed well. The working process of the whole equipments is completed cooperatively by the electric control system and the mechanical hydraulic system, and the working loads can be up to $100 \mathrm{MPa}$.

\section{The finite element analysis of hinge beam}

\subsection{Finite element model}

Because the hinge beam is the main bearing component of the cubic press. In view of the actual working conditions, this paper employs ANSYS, a kind of the analysis software of finite element, to carry out the static analysis and calculation and to explore the stress distribution of the hinge beam [6]-[8]. To facilitate the structure discretization and element analysis and keep the higher calculation accuracy, we has simplified the model [9]: for one thing, the smooth holes take the place of the threaded holes; for another, the chamfers are omitted on the upper and the lower tables. And then we establish directly the 3D solid model by ANSYS.

The selected elements are related to the properties of the tetrahedral structure module Solid186 that is a 3D 20 nodes element, which is used to establish the mesh dividing model of hinge beam. Due to the structure symmetry of hinge beam, and also its internal loads are uniform and symmetric, as it is shown in the Figure 3 that we only need to draw the mesh model of the $1 / 4$ hinge beam. The whole structures are made of the alloy structural steel of 35CrMo made in China.

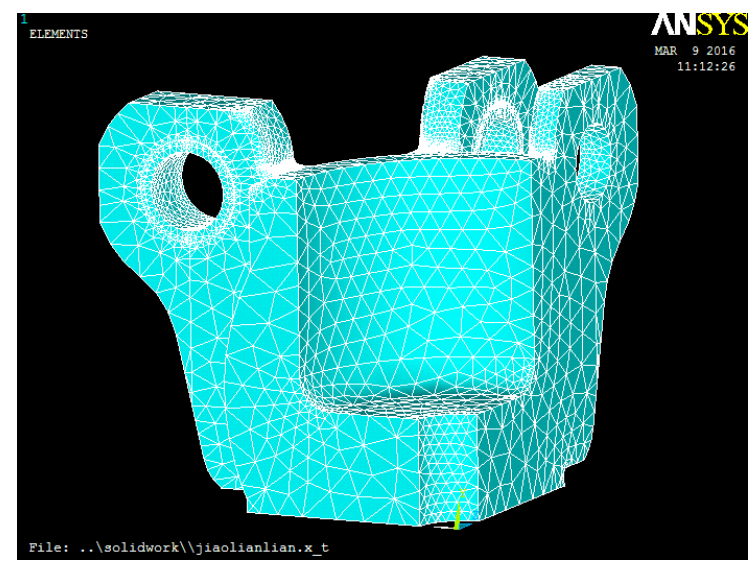

Figure 3. The mesh dividing model of the hinge beam

\subsection{Adding constraints and loads}

The hinged cubic press is the circulatory working equipment including three stages: loading, holding pressure and unloading on the condition of the high temperature and high pressure. Taking the test experiments into account this paper merely aims to simulate and analyze at the stage of holding pressure.

In order to ensure the consistency between the calculation results and the actual working situations, the method adding gradual the quantitative load to the hinge beam is applied to the whole analysis process when we use ANSYS to simulate and analyze. And assuming each lug to be in the uniform stress, the surface constraints are exerted correspondingly over the bottom of hinge beam and the symmetric cross-section of a quarter of the model. In the same way, the surface loads are exerted correspondingly over the lugholes and the bottom of hinge beam.

\subsection{Result analysis}

In this paper, we establish the static analysis model of $1 / 4$ hinge beam by PCG(Precondition Conjugate Gradient). In the light of the limited space of this article, as it is indicated in the Figure 4 that we merely draw the equivalent stress distribution nephogram of the $1 / 4$ hinge beam on the condition of $100 \mathrm{MPa}$. It is analyzed that the maximum tensile stress mainly concentrates on the upper surface of the lug roots connecting the outer cylinder with 
the both lugs. Its value is about $324.8 \mathrm{MPa}$. Meanwhile, the stress value is only $5.32 \mathrm{MPa}$ in the middle of the two lug roots and below the two lug roots. In addition, considering the safety factor of 2 2.4 in the design of hinge beam and often normal-load operation of hinge beam in the working process, so it has enough strength. However, it can be seen in the Fig. 3 that the gradients of the stress focusing on the range of 5 millimeter between the lug roots and the upper surface are larger. According to the test data, it has preliminarily verified that the results obtaining from the finite element analysis are correct and consistent.

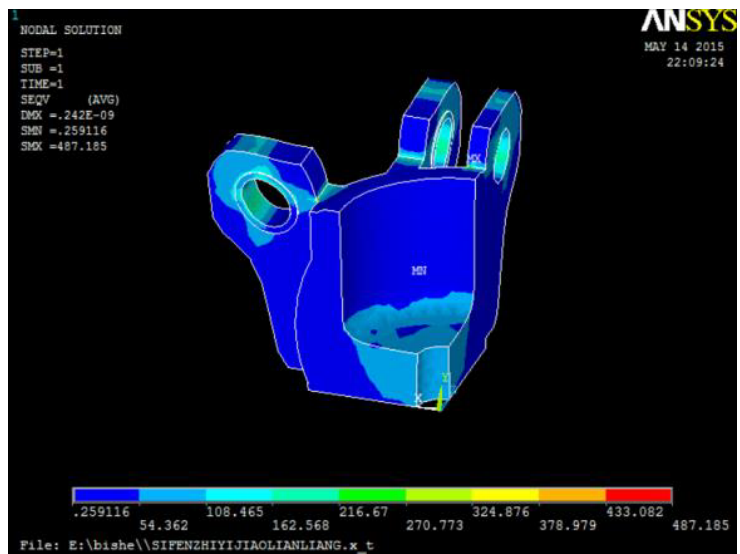

Figure 4. Von mises stress of the hinge beam

\section{Experimental verification}

\subsection{Layout schemes of the strain gauges}

According to the results of finite element analysis and the actual fracture position of the lugs, the two strain gauge groups defining the distance as 1 millimeter and consisting of 8 coaxial and multi-gate strain gauges in which the distance is designed as 3 millimeter are pasted in the lugs. So we can measure accurately the strain variation at those areas of the larger strain gradient. Additionally, in order to compare and make the test data more accurate, we choose respectively a lug to measure in the 4 directions of the two lugs and the three lugs.

\subsection{Experimental tests and result analysis}

We use the Wheatstone bridge circuit to measure the change of resistance strain gauge. Regarding $10 \mathrm{MPa}$ as the unit load increment, A successful and complete test is implemented by way of gradual loading from $10 \mathrm{MPa}$ to the maximum load $100 \mathrm{MPa}$. Moreover, after each quantitative loading, the measurement of holding pressure for 3 minutes is carried out on the hinge beam so as to ensure the stability and reliability of the test data.

After the repeated tests of loading, holding pressure and unloading, we has gotten a set of experimental data. In consideration of the limited space of this article, as it is respectively shown in the Table 1 that we only list the related data of 8 test points on the condition of $100 \mathrm{MPa}$ about GF group of a hinge beam. It is concluded from Table1, that GF5 is the point of the maximum tensile stress $311.85 \mathrm{MPa}$ which is less than the yield limit of the selected material. Comparing the test data of the upper surface of the lug roots on the condition of $100 \mathrm{MPa}$ with the data of the finite element simulation, the relative error $4.18 \%$ between the test values and the simulation and calculation values verifies the fact that the both are consistent each other. It justifies the establishment and analysis of the finite element model is reasonable and accurate.

Table 1. The related data of 8 test points in GF group

\begin{tabular}{clllllllll}
\hline \multirow{2}{*}{$\begin{array}{c}\text { Loads } \\
\text { [Mpa] }\end{array}$} & GF1 & GF2 & GF3 & GF4 & GF5 & GF6 & GF7 & GF8 \\
\hline 10 & 67 & 82 & 150 & 178 & 203 & 162 & 151 & 144 \\
20 & 120 & 144 & 264 & 309 & 355 & 292 & 273 & 260 \\
30 & 163 & 198 & 366 & 432 & 513 & 409 & 384 & 365 \\
40 & 209 & 253 & 477 & 559 & 658 & 530 & 499 & 476 \\
50 & 252 & 308 & 585 & 693 & 801 & 649 & 612 & 585 \\
60 & 294 & 359 & 685 & 798 & 940 & 764 & 721 & 687 \\
70 & 335 & 409 & 784 & 905 & 1069 & 872 & 825 & 788 \\
80 & 380 & 465 & 891 & 1035 & 1210 & 992 & 938 & 897 \\
90 & 424 & 517 & 991 & 1168 & 1345 & 1106 & 1048 & 1002 \\
100 & 463 & 566 & 1088 & 1296 & 1485 & 1215 & 1151 & 1098 \\
\hline
\end{tabular}

\section{Summary}

In this paper, we consider the hinge beam of hinged cubic press as the research subject. First, the distribution and the value size of the internal stress of hinge beam are obtained by way of employing ANSYS to carry out the static analysis and calculation of hinge beam. Consequently, The simulation results demonstrate that the internal stress of hinge beam mainly concentrates on the upper surface of the lug roots connecting the outer cylinder with the both lugs. And the stress gradients are larger in these areas. So it is more likely to generate the fracture if the lug roots appear the defects in the process of manufacturing. Furthermore, based on the experiment methods of electrical stress measurement, we have a list of the actual tests in the lug roots of hinge beam. The results show that the maximum tensile stress $311.85 \mathrm{MPa}$ is located at the position GF5. Compared to the data of finite element analysis, the relative error $4.18 \%$ between the test results and the simulation ones verifies the fact that the establishment and analysis of the finite element model is reasonable and accurate. Moreover, this paper provides a referential bases for the optimization of the following structures.

\section{Acknowledgements}

The research in this paper is funded by Innovation Project of GUET Graduate Education under Grant No.YJCXS201508 and National Students' Project for Innovation and Entrepreneurship Training Program under Grant No. 201510595001 and the Based Capacity Promotion Project of Middle-aged and Young Teachers 
in Colleges and Universities of GuangXi in 2016 under Grant No. ky2016YB147.

\section{References}

1. M.JI. DaiQi-ke,Chinese Journal of Theoretical and Applied Mechanics,Modern situations of electrical measuring and stress technology,3(2). 97-110, (1959).

2. J.Z. Nong,Z.S.Xie, Y.Wu, Superhard Material Engineering, The failure analysis on the hinge beam of the diamond synthesis cubic press, 23(6),5-9, (2011).

3. Z.R.Li,C.X.Wen,Diamond\&Abrasives Engineering, The hydraulic system failure analysis of the cubic press,3(93),36-38, (1996).

4. L.J. Huang,D.B. Cen,Modern Manufacturing Engineering,Stress-test and finite element analysis for the giant-type hydraulic vulcanizing machine, (7),126-129, (2010).
5. C. Zhang,Jilin University, The analysis and optimum design of China-type cubic high-pressure apparatus by FEM, (2010).

6. Q.G. Han, X.P. Jia, H.A. Ma, Acta Physica Sinica, Finite element simulations of thermal-stress on carbide anvil used in cubic high pressure cemented tungsten apparatus,58(7),4812-4816, (2009).

7. D.C. Qin,Y. Liang, L.P. Chen, Journal of Mechanical Strength ,Research for hinge SLEEVE'S structural optimal design based on fatigue intensity, 28(2),306310, (2006).

8. C.S. Baviskar,R.M. Tayade, V. G. Patil, Infernahonal Journal of Mechanical Engineering and Robofics Research,Determination of failure strength of curved plate weld joint using finite element analysis, 1(2012).

9. X. Sun,B. Wang,Y. Zhang,Journal of Guilin University of Technology,Hinge sleeve strength calculation in cubic press,33(2),322-324, (2013). 